\title{
THE SELECTIVE SIGNIFICANCE OF PARTIAL APOMIXIS
}

\author{
ALAN B. HARPER \\ Department of Zoology, NJ-15, University of Washington, Seattle 98195, U.S.A.
}

Received 14.vii.81

\begin{abstract}
SUMMARY
A one-locus model for the evolution of partial apomixis and the sex ratio in hermaphrodites is investigated. This model is used to find the conditions under which increased apomixis is advantageous, and to show how these conditions depend on the average sex ratio of the population. Two conceptually distinct advantages for apomictic reproduction can be discriminated: an advantage through increased rate of growth, and an advantage through non-reciprocal fertilization. A mutant that trades sperm and egg production for apomixis without changing the sperm/egg ratio will increase according to the first advantage, while a mutant that increases apomixis by decreasing egg production alone will increase according to the second advantage.

It is possible to find a frequency-dependent property of phenotypes in this model that acts much like phenotypic fitness. However, the model has some qualities that are quite different from standard one-locus selection. The phenotype of the rare homozygote can influence whether a mutation can invade a population, and Hardy-Weinberg proportions are often violated. Unlike the evolution of the sex ratio in purely sexual species, there is no quantity that is maximized by evolution in this model.
\end{abstract}

\section{INTRODUCTION}

APOMICTIC reproduction has been shown to have an intrinsic selective advantage in many situations. Analysis of this problem has concentrated on two major issues, classifying the advantages of apomixis and evaluating the magnitude of these advantages. The realization of this intrinsic advantage has stimulated discussion of the possible counter-balancing disadvantages of apomixis (Williams, 1975; Maynard Smith, 1978; Lloyd, 1980).

Maynard Smith (1971) showed one advantage apomicts have over sexuals. He compared two populations: one sexual, dioecious and 50 per cent males, the other apomictic and all-female. If sexual and apomictic females have the same number of offspring of the same viability, the apomictic population (producing only daughters) will have twice the intrinsic rate of increase of the sexuals. This two-fold advantage results from competition between the two reproductively-isolated populations. It is present regardless of the method of inheritance of apomixis, because the populations are reproductively isolated. However, the magnitude of this advantage through an increased intrinsic rate of growth is dependent on the sexuals' sex ratio.

Taylor (1979), Charlesworth (1980) and Lloyd (1980) show a different advantage to apomixis. In these models sexual and apomictic individuals produce the same number of eggs and pollen. Of course, only the sexuals' offspring incorporate genes from the pollen. The advantage of apomixis in this model is dependent both on the apomicts' frequency and on the genetics of apomixis. For instance, if apomixis is cytoplasmically inherited, it will 
not have any intrinsic advantage. Charlesworth (1980) calls this disadvantage to sexuality the "cost of meiosis", noting that the apomicts' advantage is independent of the sex ratio of the sexual population. I prefer to call this an advantage through non-reciprocal fertilization, since the meaning of "the cost of meiosis" has been subject to various interpretations (Williams, 1975; Manning, 1976; Triesman and Dawkins, 1976).

The models that have led to the identification of the advantages of apomixis are of two sorts. Some assume a single locus controls whether an organism is (obligately) sexual or apomictic (Maynard Smith, 1971; Taylor, 1979; Charlesworth, 1980; Lloyd, 1980). Others allow partial apomixis but do not include a well-defined genetic mechanism to control the expression of this trait (Williams, 1975; Lloyd, 1980). The latter evaluate the progress of selection using a phenotype's contribution to future generations.

The model I present assumes that alleles at a single locus can affect both the pollen-ovule ratio of an organism and the percentage of eggs which are apomictic. This model is used to answer these questions: What is the advantage of apomictic reproduction among hermaphrodites? Does selection on the degree of apomixis interact with selection on the pollen-ovule ratio of a population? Can something like "fitness" be ascribed to genotypes in a way that adequately describes the course of selection?

It is important to explore these factors since partial apomixis is not uncommon. Nygren (1954) and Fryxell (1957) list many partially-apomictic plant species. Knox (1967) found two grass species which varied the degree of apomixis in response to environmental factors. In other species partial apomicts have been produced by interspecific crosses between obligate apomicts (as pollen donors) and obligate sexuals (Clausen, 1954; Muntzing, 1958). This suggests that partial apomixis may have been a transitional state in these, and perhaps other, species. The latter experiments have shown oligogenic inheritance of the degree of apomixis, the following model would be appropriate to these species if each of the alleles affecting reproduction were fixed at different times.

\section{THE MODEL}

A simple model for the effects of partial apomixis is one in which one locus with two alleles controls the investment in reproduction. This model is similar to many others developed in order to investigate the evolution of the sex ratio. See Uyenoyama and Bengtsson (1979) for a discussion of these models.

The organisms are diploid, so there are three genotypes- $A A, A B$, and $B B$ - which are called genotypes one, two, and three. Generations do not overlap and sexual gametes combine at random. The organisms are hermaphrodites and the three genotypes differ only in their gamete production. Any individual can produce three sorts of gametes: haploid sperm (or pollen), haploid eggs (meiotic ovules), and apomictic diploid eggs (mitotic ovules). The latter gametes I call "apo-eggs". An adult of genotype $i$ invests $m_{i}$ in sperm production, $f_{i}$ in eggs, and $a_{i}$ in apo-eggs. It is unimportant what units are used to measure this investment; all that is necessary is that 
the number of gametes produced be directly proportional to the investment in that type of gamete.

These assumptions allow mutations that cause many different types of re-apportionment of resources among the three gamete types. Lloyd (1980) and Charlesworth (personal communication) believe that only mutations that re-allocate resources between eggs and apo-eggs are important in the evolution of hermaphroditic plants. This concern is well illustrated by pseudogamous apomicts which necessarily retain pollen production; and this broadening of the scope of the model adds mathematical complexity. However, the pollen-ovule ratio of plants is subject to evolution (Cruden, 1977), and it is certainly possible that mutations affecting it are not separate from those affecting apo-egg production. Only by examining both these effects can their interactions be understood. Unfortunately, the resulting model becomes so complicated that other factors, such as self-fertilization (Charlesworth, 1980), cannot be considered.

The derivation of the transformation equations will be more lucid if a few redundant parameters are introduced. The parameters that actually affect the population genetics are listed in table 1 . Assume that one unit

TABLE 1

The meaning of the important symbols used in the equations of the text

Symbol

$A A, A B, B B$

$x_{1}, x_{2}, x_{3}$

$p$

$m_{i}, f_{i}, a_{i}$

$\bar{m}, \bar{f}, \bar{a}$

$k=k_{1} k_{2}$

$\phi$

$l_{1}, l_{2}$
Meaning

The three diploid genotypes (genotypes 1, 2, and 3).

The genotype frequencies, primed $\left(x_{i}^{\prime}\right)$ for the next generation.

The frequency of allele $A\left(p=x_{1}+x_{2} / 2\right)$.

The investment of genotype $i$ in sperm, eggs and apo-eggs, respectively.

The average investment in those gametes $\left(\bar{m}=\sum_{i} m_{i} x_{i}\right)$.

The relative efficiency of reproduction through apo-eggs versus sexual eggs.

The proportion of egg investment out of sexual investment $[\phi=f /(m+f)]$.

Numbers proportional to the eigenvalues of the Jacobian matrix at an equilibrium.

of investment in reproduction can produce $S$ sperm, $E$ eggs, or $k_{1} E$ apo-eggs. A not-yet-fertilized sexual egg has probability $\pi$ of surviving to adulthood, an apo-egg has probability $k_{2} \pi$ of surviving. The parameter $k_{2}$ takes into account both the sexual egg's risk of not being fertilized, and any viability differences between sexually produced zygotes and apo-eggs. There are no other differences between apomictically and sexually produced individuals. This means that if two organisms have the same genotype they will have the same adult phenotype-even if one was produced sexually and the other apomictically.

These considerations limit the model to describing agamospermous apomixis, since offspring produced by vegetative apomixis do not have the same dispersal pattern as seeds. For this reason I will use "apomixis" as a synonym of "agamospermy" throughout this paper. I have not assumed anisogamy, nor that sexual eggs and apo-eggs cost the same to produce. The model is applicable to pseudogamous apomicts if the apo-eggs' probability of being fertilized, which affects $k_{2}$, does not depend on the frequency of the different phenotypes in the population.

Assume that at some time there are $N$ adults alive, and the frequencies of the three genotypes $A A, A B$, and $B B$ are $x_{1}, x_{2}$, and $x_{3}$. The frequency 
of the $\boldsymbol{A A}$ adults in the next generation will then be:

$$
x_{1}^{\prime}=\frac{\frac{\left(N x_{1} m_{1}+N x_{2} m_{2} / 2\right) S}{\sum N x_{i} m_{i} S} \pi\left(N x_{1} f_{1}+N x_{2} f_{2} / 2\right) E+k_{2} \pi N x_{1} a_{1} k_{1} E}{\sum\left(\pi N x_{i} f_{i} E+k_{2} \pi N x_{i} a_{i} k_{1} E\right)} .
$$

Simplifying and letting $\bar{m}=\sum x_{i} m_{i}, \bar{f}=\sum x_{i} f_{i}, \bar{a}=\sum x_{i} a_{i}$, and $k=k_{1} k_{2}$, we have as genotype frequencies in the next generation:

$$
\begin{aligned}
x_{1}^{\prime}= & \left\{\left(m_{1} x_{1}+m_{2} x_{2} / 2\right)\left(f_{1} x_{1}+f_{2} x_{2} / 2\right) / \bar{m}+k x_{1} a_{1}\right\} /(\bar{f}+k \bar{a}) \\
x_{2}^{\prime}= & \left\{\left(m_{1} x_{1}+m_{2} x_{2} / 2\right)\left(f_{3} x_{3}+f_{2} x_{2} / 2\right) / \bar{m}\right. \\
& \left.+\left(m_{3} x_{3}+m_{2} x_{2} / 2\right)\left(f_{1} x_{1}+f_{2} x_{2} / 2\right) / \bar{m}+k x_{2} a_{2}\right\} /(\bar{f}+k \bar{a}) \\
x_{3}^{\prime}= & \left\{\left(m_{3} x_{3}+m_{2} x_{2} / 2\right)\left(f_{3} x_{3}+f_{2} x_{2} / 2\right) / \bar{m}+k x_{3} a_{3}\right\} /(\bar{f}+k \bar{a}) .
\end{aligned}
$$

The meaning of the parameters in these equations is summarized in table 1. Because $x_{1}^{\prime}+x_{2}^{\prime}+x_{3}^{\prime}=1$, any pair of equations (1) will define the behaviour of the model.

Notice that the values of $S$ and of $E$ have cancelled in these equations. This means that it is only the investment in sexual gametes, not the number of gametes produced, that affects the population genetics. The same result is seen in purely sexual systems (Kolman, 1960). The parameter $k=k_{1} k_{2}$ measures the number of individuals produced by one unit invested in apo-eggs compared to the number produced by one unit in sexual eggs.

The population size $N$ also cancels out of these equations. The population size in the next generation is the number of sexual and apomictic zygotes that survive:

$$
N^{\prime}=(\pi E \bar{f}+k \pi E \bar{a}) N
$$

\section{CONSTANT SEX RATIO, CONSTANT TOTAL INVESTMENT}

If each genotype has the same total investment in reproduction and the same sex ratio, then the behaviour of the model is greatly simplified. Let genotype $i$ invest $a_{i}$ in apo-eggs and $\left(1-a_{i}\right)$ in sexual gametes. The investment in sex is divided into $f_{i}=\phi\left(1-a_{i}\right)$ into eggs, and $m_{i}=(1-\phi)\left(1-a_{i}\right)$ into sperm. The parameter $\phi$ measures the proportion of investment in egg production out of all investment in sexual gametes. This means that for each unit invested in sexual gametes (eggs plus sperm), $\phi \pi E$ adult offspring are produced; while for each unit invested in apomixis, $k \pi E$ are produced. Under these conditions, sexual reproduction is more efficient than apomixis when $k<\phi$.

The behaviour of the model with constant sex ratio can be approximately described thus: If apomixis is more efficient than sexuality $(k>\phi)$ then the genotype investing the most in apomixis is most fit, if apomixis is less efficient $(k<\phi)$ this genotype is least fit, and if $k=\phi$ there is no change in the gene frequency. (This rule is not exactly correct, the exception to it is discussed in section 4.) The advantage of apomixis here depends on the (constant) sex ratio; this result is similar to that found by Lloyd for dioecious species. Sex-ratio theory predicts that $\phi=0.5$ in many panmictic sexual 
species. If this is so, then apomixis can be said to have a two-fold advantage since a mutant gene that trades two successful meiotic offspring for one mitotic offspring will not change in frequency.

Fig. 1 illustrates the behaviour of the model for various values of the parameters. It shows that the gene frequency changes are in accord with this rule; for instance, when the heterozygote is more fit according to the rule (fig. 1a) there is a central stable equilibrium. It also illustrates the degree to which the population can diverge from Hardy-Weinberg proportions even when the "fitnesses" of the genotypes are equal (fig. 1f). The position of the central equilibrium (when it exists) can be found explicitly.

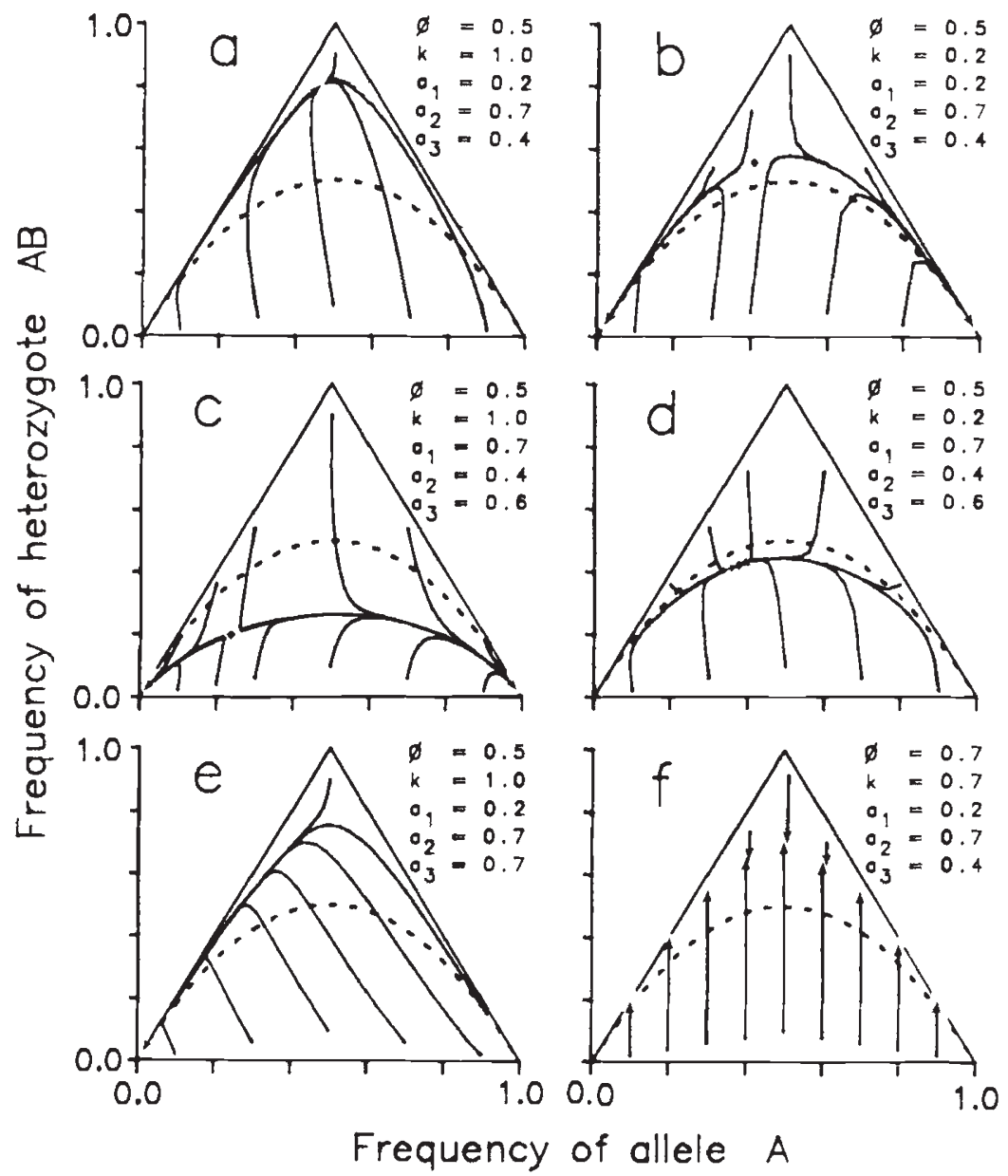

FIG, 1.-The behaviour of the model with constant sex ratio and various values of the parameters. The meaning of the parameters is given in table 1 . The solid lines show the evolution of gene frequencies from various initial conditions, and the dashed line connects Hardy-Weinberg points. The cross marks the polymorphic equilibrium, when it exists. Figs. (a) and (d) show behaviour qualitatively like overdominance, (b) and (c) like underdominance. In (e) the dominant allele $B$ is more fit than $A$, in (f) the gene frequencies do not change. In all cases, the behaviour follows the rule given in section 3 . 


\section{RARE-ALLELE ANALYSIS}

The transformation equations (1) can have a number of different equilibria, and it is impossible to find and characterize them all. The local stability conditions of the two trivial equilibria $x_{1}=1$ ( $A$ fixed) and $x_{3}=1$ ( $B$ fixed) can be found rather easily. In Appendix $A$ it is shown that a necessary condition for the equilibrium $x_{1}=1$ to be stable to invasion is $l_{1}<0$ and $l_{2}<0$, where

$$
\begin{aligned}
& l_{1}=\left(f_{2}-f_{1}\right) m_{1}+\left(m_{2}-m_{1}\right) f_{1}+2 k m_{1}\left(a_{2}-a_{1}\right) \\
& l_{2}=k a_{3}-k a_{1}-f_{1} .
\end{aligned}
$$

(These values have the same sign as the eigenvalues of equations (1) at this equilibrium.) The stability equations for the equilibrium $x_{3}=1$ are the same as those for $x_{1}=1$, except that the subscripts 1 and 3 are interchanged.

Equation (4b) shows that the stability of a population to invasion by a mutant allele is influenced by the phenotype of the rare homozygote. Such behaviour is not seen in models where the genotypes differ by fitness differences alone. This is the major caveat to the rule in section 3 that describes evolution with a constant sex ratio and constant total investment. One cannot exactly describe the evolution of the two allele model by assigning a fitness to each of the three genotypes independently of the others.

The rule in section 3 would imply that if $k$ is greater than $\phi$, and if the heterozygote invests less in apomixis than either homozygote, then underdominance behaviour will be seen and both fixed states will be locally stable. In fact, one of the fixed states can be unstable under these conditions. The behaviour can occur $\left(l_{2}>0\right)$ only when the rare homozygote produces more offspring through apo-egg production than the common homozygote produces by sexual and apomictic egg production together.

This effect should be important under only two conditions. The population size must be large enough for mutation and recombination to produce the rare homozygote even with selection against the heterozygote. And the rare homozygote must invest more in apomixis than the heterozygote $\left(a_{3}>a_{2}\right)$. (If $a_{3} \leqq a_{2}$ it can be shown that $l_{2}>0$ implies that $l_{1}>0$.) This means that the value of $l_{2}$ will not affect the stability behaviour of dominant mutations.

\section{AN EVOLUTIONARILY STABLE STRATEGY}

An evolutionarily stable strategy (ESS) is a rule such that if it is followed by all members of a population, no rule-breaking rare heterozygotic mutant can increase in frequency (Maynard Smith, 1976). In this model an ESS is a rule ensuring that $l_{1} \leqq 0$ for all possible phenotypes. The form of an ESS in this model depends on the range of phenotypes that mutation can generate.

As one example, suppose that mutations affecting the sex ratio do not necessarily affect the degree of apomixis and vice-versa, and that total investment in reproduction is constant. (The latter condition is not as restrictive as it might seem. Let $M_{i}, F_{i}$, and $A_{i}$ be the numbers of sperm, eggs and apo-eggs produced by a certain phenotype. The "constant total 
investment" criterion says merely that there are constants $S, E$, and $k_{1} E$ such that, for all possible phenotypes

$$
M_{i} / S+F_{i} / E+A_{i} /\left(k_{1} E\right)=1 \text {. }
$$

This is true because, as stated in the introduction, the units in which investment is measured and the value of the constants $S$ and $E$ are irrelevant to the behaviour of the model. Letting $k_{2}$ be the relative viability of apo-eggs as compared to sexual eggs, $k=k_{1} k_{2}$ recovers $k$ as used in equations (1).)

Suppose that the heterozygotic phenotype has the same sex ratio as the homozygote, but invests a different amount in apomixis. Then, as in section $3, m_{i}=(1-\phi)\left(1-a_{i}\right)$ and $f_{i}=\phi\left(1-a_{i}\right)$, and

$$
l_{1}=C(k-\phi)\left(a_{2}-a_{1}\right),
$$

where $C$ is always greater than 0 .

Suppose instead that the two phenotypes have different sex ratios but invest the same in apomixis, then $a_{1}=a_{2}$ which implies $m_{1}+f_{1}=m_{2}+f_{2}$ and

$$
\begin{aligned}
l_{1} & =m_{1}\left(f_{2}-f_{1}\right)+f_{1}\left(m_{2}-m_{1}\right) \\
& =\left(m_{1}-f_{1}\right)\left(f_{2}-f_{1}\right) .
\end{aligned}
$$

This equals zero for all possible values of $f_{2}$ when $m_{1}=f_{1}$; while there are phenotypes that can invade if $m_{1} \neq f_{1}$.

It can be seen that the ESS that ensures that $l_{1} \leqq 0$ is: Invest equally in sperm and eggs $\left(m_{1}=f_{1}\right.$, or $\left.\phi_{1}=0.5\right)$; invest as much as possible in apomixis if apo-eggs are more than one-half as efficient as sexual eggs (i.e., if $k>0.5$ ), invest as little as possible if they are less than one-half as efficient. This ESS holds only if changes in the sex ratio and the degree of apomixis can occur independently and if all trade-offs between producing the three types of gametes are linear. Under other conditions other ESS's will obtain.

In a purely sexual system with panmixis a strong characterization of the ESS can be found: No matter what constraints evolution enforces, maximize $m \times f$ (the number of sperm times the number of eggs produced) given those constraints (MacArthur, 1965; Maynard Smith, 1978).

This is the ESS because any phenotype with a higher value of $m \times f$ can invade a population made up of a phenotype with a lower value of $m \times f$. It can be shown that for partial apomicts there is no function of $m, f$, or $a$ that has this ranking property. So there is probably no characterization of the ESS of a partially-apomictic system under a general phenotypic constraint. There is also no phenotypic quantity maximized by evolution.

\section{A PHENOTYPIC MODEL}

There is one important conclusion about the evolution of a partiallyapomictic population that cannot be reached using a one-locus model. The relative fitnesses of phenotypes investing different amounts in sperm, eggs, and apo-eggs are frequency dependent, so that potential evolutionarilystable states can often be simply characterized. Under such conditions evolution should tend towards states where all phenotypes have equal fitness, if there is sufficient genetic flexibility to allow such states (Slatkin, 1978). 
In a partially-apomictic hermaphroditic population, the most reasonable measure of a phenotype's fitness is its genetic representation in the next generation. This is the number of apomictically-produced offspring plus one-half the number of sexually-produced offspring. To find this fitness, let a certain individual invest $m_{i}, f_{i}$, and $a_{i}$ in sperm, eggs, and apo-eggs, respectively. Its genetic representation in the next generation is then

$$
w_{i}=\left[(\bar{f} / \bar{m}) m_{i}+f_{i}+2 k a_{i}\right] \pi E / 2
$$

where $k$ is defined in table 1 , and the $\bar{f}$ and $\bar{m}$ are averages over all phenotypes. This function has two properties that convince one that it is a valid measure of fitness. First, the average fitness of the population is

$$
\bar{w}=\pi E(\bar{f}+k \bar{a})
$$

which is the average number of reproducing offspring per individual (see equation (2)). Second, a rare heterozygotic phenotype will increase in frequency just when its fitness is greater than average (i.e., $w_{i}>\bar{w}$ when phenotype $i$ is rare implies that $l_{1}>0$ ).

In the introduction it was noted that there are two sorts of advantages an invading apomict can have: either an advantage through higher growth rate (if the invading apomict diverts energy from sexual reproduction to apo-egg production and retains the sexual's sex ratio); or an advantage through non-reciprocal fertilization (if the invader diverts energy from sexual egg production to apo-egg production). These advantages can now be compared.

Assume that the original population, consisting of phenotype 1 , invests $a$ in apo-egg production, $\phi(1-a)$ in egg production and $(1-\phi)(1-a)$ in sperm production. Two sorts of mutants might arise. The first invests $a+\Delta a$ in apo-egg production, but retains the sex ratio of phenotype 1 . This mutant can invade when $k>\phi$. There is an advantage to apomixis through a higher growth rate only when apomictic reproduction is more efficient than sexual reproduction, and this will depend on the value of $\phi$. The other mutant sacrifices egg production for apo-egg production, so that it invests $a+\Delta a$ in apo-eggs and $\phi(1-a)-\Delta a$ in eggs. It can invade when $k>0.5$. There is an advantage to apomixis through non-reciprocal fertilization when apo-eggs are more than one-half as likely to survive as sexual eggs, and this is independent of $\phi$.

This parallels Charlesworth's (1980) results. He shows that an obligately apomictic female in a dioecious species has an advantage that depends on the sexual's sex ratio (assuming no male investment in offspring). While a hermaphrodite that produces apo-eggs and sperm has an advantage that is independent of the sex ratio. Thus there are two conceptually distinct advantages of apomictic reproduction: an advantage of higher growth rate, and an advantage of non-reciprocal fertilization. If the advantage of apomixis is measured by the minimum value of $k$ that favours apomixis, then the two advantages are equal when investment in eggs and sperm is equal $(\phi=0 \cdot 5)$.

Acknowledgments. - I would like to thank M. Slatkin and J. Felsenstein for help and encouragement throughout the development of these ideas. I also thank J. Waage for stimulating my interest in evolution, and in this problem. M. Kirkpatrick and B. Charlesworth criticized manuscript copies of this paper. 


\section{REFERENCES}

CHARLESWORTH, B. 1980. The cost of sex in relation to mating system. Journal of Theoretical Biology, 84, 655-671.

Clausen, J. 1954. Partial apomixis as an equilibrium system in evolution. Proceedings of the Ninth International Congress of Genetics, pp. 469-479.

CRUDEN, R. W. 1977. Pollen-ovule ratios: a conservative indicator of breeding systems in flowering plants. Evolution, 31, 32-46.

FRYXELL, P. A. 1957. Mode of reproduction of higher plants, Botanical Review, 23, 135-233.

KNOX, P. B. 1967. Apomixis: seasonal and population differences in a grass. Science, 157 , 325-326.

KOLMAN, W. A. 1960. The mechanism of natural selection for the sex ratio. American Naturalist, 94, 373-377.

LLOYD, D. G. 1980. Benefits and handicaps of sexual reproduction. Evolutionary Biology, $13,69-111$.

MACARTHUR, R. H. 1965. Ecological consequences of natural selection. In Theoretical and Mathematical Biology, eds. T. H. Waterman and H. J. Morowitz, pp. 388-397. Blaisdell, New York.

MANNING, J. T. 1976. Gamete dimorphism and the cost of sexual reproduction: are they separate phenomena? Journal of Theoretical Biology, 55, 393-395.

MAYNARD SMITH, J. 1971. The origin and maintenance of sex. In Group Selection, ed. G. C. Williams, pp. 163-175. Aldine Atherton, Chicago.

MAYNARD SMITH, J. 1976. Evolution and the theory of games. American Scientist, 64, 41-45. MAYNARD SMITH, J. 1978. The Evolution of Sex. Cambridge University, New York.

MUNTZING, A. 1958. The balance between sexual and apomictic reproduction in some hybrids of Potentilla. Hereditas, 44, 145-160.

NYGREN, A. 1954. A pomixis in the angiosperms. II. Botanical Review, 20, 577-649.

SLATKIN, M. 1978. On the equilibration of fitness by natural selection. American Naturalist, $112,845-859$.

TAYLOR, P. D. 1979. An analytical model for a short-term advantage for sex. Journal of Theoretical Biology, 81, 407-421.

TRIESMAN, M., AND DAWKINS, R. 1976. The "cost of meiosis": is there any? Journal of Theoretical Biology, 63, 479-484.

UYENOYAMA, M. K., AND BENGTSSON, B. O. 1979. Towards a genetic theory for the evolution of the sex ratio. Genetics, 93, 721-736.

WilliaMs, G. C. 1975. Sex and Evolution. Princeton University Press.

\section{APPENDIX A}

\section{The eigenvalues of the fixed equilibria}

The transformation equations for $x_{1}$ and $x_{3}$ [equations (1a) and (1c)] are sufficient to find the eigenvalues of the equilibrium $p=1$. Define two functions: $f_{1}\left(x_{1}, x_{3}\right)=x_{1}^{\prime}-x_{1}$, and $f_{3}\left(x_{1}, x_{3}\right)=x_{3}^{\prime}-x_{3}$. Then an equilibrium is stable when the eigenvalues of the Jacobian of $\left(f_{1}, f_{3}\right)$ are both less than zero and greater than -2 .

We have from equation (1a)

$$
\begin{aligned}
f_{1} & =x_{1}^{\prime}-x_{1} \\
& =\left[\left(x_{1} m_{1}+x_{2} m_{2} / 2\right)\left(x_{1} f_{1}+x_{2} f_{2} / 2\right) / \bar{m}+x_{1} a_{1} k\right] /(\bar{f}+k \bar{a})-x_{1}
\end{aligned}
$$

and a similar equation for $f_{3}$. These can be differentiated once it is noted that $\partial x_{2} / \partial x_{1}=\partial x_{2} / \partial x_{3}=-1$, because $x_{2}=1-x_{1}-x_{3}$. We find that at $x_{1}=1$ (and $\left.x_{3}=0\right) \quad \partial f_{3} / \partial x_{1}=0$, so that the eigenvalues $\lambda_{1}$ and $\lambda_{2}$ of 
the Jacobian matrix are these two partial derivatives evaluated at the equilibrium

$$
\begin{aligned}
\lambda_{1} & =\partial f_{1} / \partial x_{1} \\
& =\left[m_{1} f_{2}+m_{2} f_{1}-2 m_{1} f_{1}+2 k m_{1}\left(a_{2}-a_{1}\right)\right]\left(2 m_{1}\right)^{-1}\left(f_{1}+k a_{1}\right)^{-1} \\
\lambda_{2} & =\partial f_{3} / \partial x_{3}=\left(k a_{3}-k a_{1}-f_{1}\right) /\left(b_{1}+k c_{1}\right) .
\end{aligned}
$$

These eigenvalues are, except for some always-positive multiplicands, the values of $l_{1}$ and $l_{2}$ reported in the text. I only discuss $l_{1}$ and $l_{2}$ in this paper because I do not feel that oscillatory instability behaviour $\left(\lambda_{1}<-2\right.$ or $\lambda_{2}<-2$ ) has much biological relevance for this model. If, in fact, parameter values are observed in nature that could lead to oscillatory instability, then this behaviour should be investigated.

The eigenvectors associated with these eigenvalues are, respectively,

$$
\begin{aligned}
& y_{1}=(1,0) \\
& y_{2}=\left(1, \frac{\partial f_{3} / \partial x_{3}-\partial f_{1} / \partial x_{1}}{\partial f_{1} / \partial x_{3}}\right) .
\end{aligned}
$$

Equation (A3a) implies that if the population has only $A A$ homozygotes and $A B$ heterozygotes in it [i.e., $\left(x_{1}, x_{3}\right)=(1-c, 0)$ for some small value of $c$, then the stability of the population to invasion is governed by the value of $l_{1}$ alone. 